\title{
Kirchen und weltanschauliche Gemeinschaften im Primärrecht der EU nach dem Vertrag von Lissabon
}

von Markus Söbbeke

Der Vertrag über die Arbeitsweise der Europäischen Union (AEUV) in der Fassung des Vertrages von Lissabon übernimmt in seinem Artikel 17 die Bestimmungen des gescheiterten Verfassungsvertrages zu den Kirchen und weltanschaulichen Gemeinschaften. Damit werden diese erstmals im Primärrecht der Union erwähnt. Die Union wird verpflichtet, den sich aus dem jeweiligen nationalen Staatskirchenrecht ergebenden Status zu ,, achten “ und nicht zu ,, beeinträchtigen“. Diese Norm ergänzt die durch die Europäische Grundrechtecharta gewährte Religionsfreiheit. Darüber hinaus ist die Union zum Dialog mit den Religionsgemeinschaften verpflichtet. In allen Fällen gilt jedoch, dass die Definition von Religionsgemeinschaften nicht Sache der Union ist, sondern diese sich nach dem jeweiligen nationalen Recht richten muss.

Art. 17 of the Treaty on the Functioning of the European Union according to the Treaty of Lisbon adopts the provisions on churches and non-confessional organisations of the failed Constitutional Treaty. Religious communities will thus be mentioned for the first time in the Union's primary law. Thereby, the Union has to "respect" and not to "prejudice" their status according to national law. This clause complements the right of religious freedom granted by the European Charter of Fundamental Rights. Furthermore, the Union is being committed to a dialogue with the religious communities. In any case, however, the definition of a religious community does not lie within the Union's competence but has to follow the given national legislation.

Der Vertrag über die Arbeitsweise der Europäischen Union (AEUV) in der Fassung des Vertrages von Lissabon enthält im Artikel 17 die erste Erwähnung von Kirchen, Religions- und Weltanschauungsgemeinschaften in der Sphäre des Primärrechts der EU. ${ }^{1}$ Diese Norm ist von hohem Interesse im Zusammenhang mit der Rechtsmaterie, die ,inzidentes Religionsrecht ${ }^{\star 2}$ der EU genannt werden

1 Der Beitrag beruht auf einer Untersuchung des Art. I-52 VVE im Rahmen der Studie Söbbeke-Krajewski, M.: Der religionsrechtliche Acquis Communautaire der Europäischen Union, Berlin, 2006.

2 Ebd., 370. 
kann. Gemeint sind damit die europarechtlichen Normen von religionsrechtlicher Relevanz, die die EU schafft, wenn ihre gesetzgeberische Tätigkeit in einem ihr zugewiesenen Kompetenzfeld eine Mitregelung religionsrechtlich relevanter Aspekte erforderlich macht. Häufig finden sie sich als Ausnahmeregeln zu Gunsten von Religionsgemeinschaften in EU-Rechtsakten. ${ }^{3}$ Das inzidente Religionsrecht der Europäischen Union entsteht auf diese Weise, ohne dass der EU eine spezifische religionsrechtliche Kompetenz zugewiesen ist.

Artikel 17 AEUV entstammt dem Vertrag über eine Verfassung für Europa (VVE), in dem er als Art. I-52 enthalten ist. Als gesicherte Substanz des Verfassungsvertrages wurde er in den Vertrag über die Arbeitsweise der Europäischen Union überführt, der die Substanz des nicht ratifizierten Verfassungsvertrages in Rechtskraft setzen soll. Gemäß Art. 17 Abs. 1, 2 AEUV achtet die Union den Status der Kirchen und der religiösen und weltanschaulichen Vereinigungen, den diese nach dem Recht ihrer Mitgliedstaaten genießen. Art. 17 Abs. 3 AEUV sieht einen offenen, transparenten und regelmäßigen Dialog der Europäischen Union mit diesen Gemeinschaften vor. Diese erstmalige Erwähnung und Anerkennung der Kirchen und Religionsgemeinschaften im Primärrecht reflektiert auch die Entwicklung der EU von einer reinen Wirtschaftsgemeinschaft zu einem ,Staatenverbund ${ }^{“ 4}$, der auf einer Wertegemeinschaft basiert. In einer Zeit der Ungewissheit über die künftige Entwicklung der Vertragsgrundlagen der EU und eines verbreiteten Unbehagens über einen als bürgerfern und demokratiedefizitär wahrgenommenen europäischen Gesetzgeber zeigt die Norm perspektivisch, wie ein erneuerter EU-Vertrag den vermissten Dialog der EU-Organe mit wichtigen gesellschaftlichen Kräften befördern und primärrechtlich absichern könnte. Die stetig wachsende Bedeutung der EU begründet die Notwendigkeit dieses Dialogs. In einer Bestandaufnahme der Rechtsmaterie von religionsrechtlicher Relevanz in der EU lässt sich demonstrieren, wie in der Evolution von einer Wirtschaftsgemeinschaft in eine rechtlich verfasste Wertegemeinschaft unausweichlich religionsrechtliche Fragen entstehen. ${ }^{5}$ Diese lassen dann auch die Religions-

3 Vgl. die Bestandaufnahme in ebd., $41 \mathrm{ff}$.

4 So der Terminus, den das BVerfG im „Maastricht-Urteil“ (BVerfGE 89, 155 ff.) gewählt hat, um die EU außerhalb der klassischen Kategorien des Völkerrechts zu charakterisieren.

5 Vgl. zur Entstehung religionsrechtlicher Fragen in der EU Söbbeke-Krajewski, M., a. a. O., $41 \mathrm{ff}$. (mit Bestandsaufnahme zur Rechtsmaterie von religionsrechtlicher Relevanz in der EU); Weninger, M.H.: Europa ohne Gott ? - Die Europäische Union und der Dialog mit den Religionen, Kirchen und Weltanschauungsgemeinschaften, Baden-Baden, 2007; zur Wertegemeinschaft EU s. a. Gaertner, J.: Die Europäische Union als Wertegemeinschaft zwischen Wettbewerbspolitik und sozialer Gestaltung, in: Informationes Theologiae Europae, 10 (2001), 153-164; spezieller zu christlichen Werten in der EU Joblin, 
und Weltanschauungsgemeinschaften als solche, d.h. als verfasste Träger von Religion und Weltanschauung, in Erscheinung treten. Die EU kann sie nicht länger nur in der Dimension wirtschaftlich handelnder Subjekte wahrnehmen. ${ }^{6}$ Umgekehrt stellt sich auch für Religions- und Weltanschauungsgemeinschaften die Frage nach einer rechtlichen Ausgestaltung ihres Verhältnisses zu der sich formenden Ordnungsgewalt auf europäischer Ebene. Die primärrechtliche Aufnahme des Art. 17 AEUV ist daher konsequent und schlüssig. ${ }^{7}$

\section{Zur Entstehungsgeschichte des Art. 17 AEUV}

\section{Ursprung in der Erklärung Nr. 11 zum Vertrag von Amsterdam}

Artikel 17 AEUV ist aus der unverbindlichen Erklärung Nr. 11 zum Vertrag von Amsterdam betreffend den Status der Kirchen und weltanschaulichen Gemeinschaften hervorgegangen. ${ }^{8}$ Der Verfassungskonvent hat die Erklärung fast wörtlich als Absätze 1 und 2 des Art. I-52 VVE übernommen und um einen dritten Absatz ergänzt. ${ }^{9}$ Die Ansichten über die genaue Bedeutung der Erklärung Nr. 11 gingen auseinander. Während einige Stimmen der Erklärung bindende rechtliche Wirkung als völkerrechtlicher Vertrag zuerkennen wollten, ${ }^{10}$ wurde sie überwie-

$J .:$ Valeurs chrétiennes et construction de l'Europe, in: Informationes Theologiae Europae, 5 (1996), 145-158; zur „Präambeldiskussion“ jüngst Naumann, K.: Eine religiöse Referenz in einem Europäischen Verfassungsvertrag, Tübingen, 2008.

6 Pointiert Muckel, S.: Die Rechtsstellung der Kirchen und Religionsgemeinschaften nach dem Vertrag über eine Verfassung für Europa, in: Die öffentliche Verwaltung, 58/5 (2005), 191-200, hier 195, der in Anlehnung an EuGH, Urteil v. 23.01.1997, Rs. C-463/93, Slg. 1997, I-255 ff. feststellt, als politische Union müsse die EU Kirchen und Religionsgemeinschaften auch in anderen Dimensionen und Funktionen wahrnehmen denn als Verpächterin von der Milcherzeugung dienenden landwirtschaftlichen Grundstücken.

7 Mückl, S.: Europäisierung des Staatskirchenrechts, Baden-Baden, 2005, 453 f., stellt fest, die normative Rangerhöhung von der bloßen Erklärung zum verbindlichen europäischen Primärrecht stehe auf der Agenda des weiteren Integrationsprozesses.

8 Vgl. dazu ausführlich Vachek, M.: Das Religionsrecht der Europäischen Union im Spannungsfeld zwischen mitgliedstaatlichen Kompetenzreservaten und Art. 9 EMRK, Frankfurt a.M. u.a., 2000, 125 ff.; Grzeszick, B.: Die Kirchenerklärung zur Schlußakte des Vertrags von Amsterdam, in: Zeitschrift für evangelisches Kirchenrecht, 48/3 (2003), 284-300 m. w. N.; Heintzen, M.: Die Kirchen im Recht der Europäischen Union, in: Isensee, J./Rees, W./Rüfer, W. (Hg.): Dem Staate, was des Staates ist - der Kirche, was der Kirche ist. Festschrift für Joseph Listl, Berlin, 1999, 29-47, hier 33 ff.

$9 \mathrm{Zu}$ deren Entstehungsgeschichte einschließlich alternativer Entwürfe vgl. Weninger, M.H., a.a.O., 98 ff.; Vachek, M., a. a. O., 125 ff.; Grzeszick, B., a. a. O., 285 ff.; Heinig, H. M.: Öffentlich-rechtliche Religionsgesellschaften, Berlin, 2003, $415 \mathrm{ff}$.

10 Robbers, G.: Partner für die Einigung: die Kirchenerklärung der Europäischen Union, in: Herder-Korrespondez, 51/12 (1997), 622-626, hier 624. 
gend und zutreffend als rechtsunverbindlicher Akt (,,soft law“) gesehen, der lediglich als Auslegungshilfe bei der Interpretation des EU-Rechts (etwa durch den EuGH) hätte Relevanz erlangen können. ${ }^{11}$ Zwar gab es bereits vor der Aufnahme in den Entwurf des VVE Bemühungen, die Bedeutung der Erklärung Nr. $11 \mathrm{zu}$ erhöhen, indem sie in Zusammenhang mit Art. 6 Abs. 3 EUV (Art. 4 Abs. 2 AEUV) gebracht wurde, nach dem die EU die nationale Identität der Mitgliedstaaten zu achten hat. ${ }^{12}$ Aber daraus kann auch in Verbindung mit der Erklärung keine Unantastbarkeit des nationalen Staatskirchenrechts als Ganzem, im Sinne einer Bereichsausnahme, hergeleitet werden. Denn erstens bedeutet „achten“ nicht, dem nationalen Recht bzw. einem Teilbereich unbedingten Vorrang einzuräumen, sondern nur, ihm den gebührenden Platz im Gefüge des Unionsrechts zu gewähren. ${ }^{13}$ Zweitens erfasst die nationale Identität i.S.d. Art. 6 Abs. 3 EUV (Art. 4 Abs. 2 AEUV) nicht das gesamte Staatskirchenrecht, sondern nur dessen Grundzüge, soweit es nämlich charakterisierender Ausdruck der nationalen Iden-

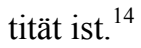

Obwohl die Erklärung Nr. 11 rechtsunverbindliches soft law ist, blieb sie nicht wirkungslos: Der europäische Gesetzgeber hat sich auf sie bezogen, um die Ausnahme des Art. 4 Abs. 2 der Gleichbehandlungsrichtlinie 2000/78/EG zu Gunsten von Kirchen und Ethos-basierten Organisationen zu rechtfertigen. ${ }^{15}$ Die Kirchenerklärung klingt auch an in der Richtlinie 2002/14/EG über die Unterrichtung und Anhörung der Arbeitnehmer in der Europäischen Gemeinschaft. ${ }^{16}$

11 Weninger, M.H., a.a.O., $96 \mathrm{ff}$.; Mückl, S., a.a.O., 454; Schnabel, P.R.: Die Stellung der Kirchen im Verfassungsvertrag der EU, in: Kirche und Recht, 9/3-4 (2003), 155-178, hier 160; Heintzen, M., a. a.O., 47; Grzeszick, B., a. a.O., 298; de Wall, H.: Neue Entwicklungen im europäischen Staatskirchenrecht, in: Zeitschrift für evangelisches Kirchenrecht, 47/2 (2002), 205-219, hier 212 m. w. N.

12 Heintzen, M., a.a.O., 47; Rüfner, W.: Religion und Kirchen vor der Europäischen Verfassung, in: Bröhmer, J. u.a. (Hg.): Internationale Gemeinschaft und Menschenrechte. Festschrift für Georg Ress, Köln u. a., 2005, 757-771, hier 769; Weber, H.: Die individuelle und kollektive Religionsfreiheit im europäischen Recht einschließlich ihres Rechtsschutzes, in: Zeitschrift für evangelisches Kirchenrecht, $47 / 2$ (2002), 265-302, hier 283; Robbers, G.: Staat und Religion, in: Veröffentlichungen der Vereinigung der Deutschen Staatsrechtslehrer, 59 (2000), 231-263, hier 257 f.; Grzeszick, B., a. a. O., 298.

13 Puttler, A.: Art. 6 EUV, Rn. 49., in: Calliess, C./Ruffert, M. (Hg.): EUV/EGV, 3. Aufl., München, 2007.

14 Walter, C.: Religionsverfassungsrecht in vergleichender und internationaler Perspektive, Tübingen, 2006, 412 ff.; Söbbeke-Krajewski, M., a. a. O., $218 \mathrm{ff.}$

15 Richtlinie 2000/78/EG des Rates vom 27.11.2000 zur Festlegung eines allgemeinen Rahmens für die Verwirklichung der Gleichbehandlung in Beschäftigung und Beruf, ABIEU Nr. L 303/16 vom 02.12. 2000, hier Erwägungsgrund Nr. 24; Mückl, S., a.a.O., S. 455; Grzeszik, B., a.a. O., 296; de Wall, H., a. a. O., 211.

16 Richtlinie 2002/14/EG des Europäischen Parlaments und des Rates vom 11.03. 2002 zur Festlegung eines allgemeinen Rahmens für die Unterrichtung und Anhörung der Arbeitnehmer in der Europäischen Gemeinschaft, ABIEU Nr. L 80/29 vom 23.03. 2002, hier Erwägungsgrund Nr. 24. 
Zweckdienlich war die Erklärung aber auch darin, die institutionelle „Sichtbarkeit“" der Kirchen, Religions- und Weltanschauungsgemeinschaften auf europäischer Ebene zu erhöhen. Zusammen mit einem gewissen Gewöhnungseffekt an die Erwähnung religiöser und weltanschaulicher Organisationen auf EU-Ebe$n \mathrm{e}^{17}$ - eine solche Erwähnung löste bei Staaten mit striktem Trennungssystem zunächst Unbehagen aus - dürfte die Erklärung erfolgreich als „Türöffner“ und Ausgangsbasis für die Bemühungen fungiert haben, den Art. I-52 VVE bzw. Art. 17 AEUV zu schaffen.

\section{Die Arbeiten des Verfassungskonvents an Art. I-52 VVE}

Aufschluss über die Bedeutung des Artikels 17 AEUV bzw. Art. I-52 VVE im Gesamtkontext des Verfassungsvertrages gibt die Behandlung in den Beratungen des „Konvents zur Zukunft Europas“, dem Verfassungskonvent, der die unverändert in den AEUV überführte Fassung erstellt hat. Der Verfassungskonvent befasste sich erstmalig in der 16. Plenarsitzung vom 27./28. Februar 2003 mit einem Entwurf der Norm. ${ }^{18}$ Konventspräsident Giscard d'Estaing hatte anlässlich der Vorstellung der Artikel 1-16 des Verfassungsentwurfs angekündigt, dass das Konventspräsidium zu einem späteren Zeitpunkt vorschlagen würde, die Erklärung Nr. 11 in den Verfassungstext zu übernehmen. ${ }^{19}$ Daraus entstand eine breit angelegte Debatte über die Funktion einer ausdrücklichen Vorschrift zum Verhältnis der EU zu den Religionsgemeinschaften. Einige Konventsmitglieder lehnten jeglichen Hinweis auf die Religion im Verfassungsvertrag ab, während andere die Bedeutung der Religionsfreiheit oder der Säkularität des Staates abstrakt betonten. Der Abgeordnete Follini unterstützte in seinem Redebeitrag ausdrücklich die Notwendigkeit einer Regelung über institutionalisierte Beziehungen zwischen Union und Religionsgemeinschaften und begründete seine Ansicht damit, dass diese Klausel es gerade erlaube, die Trennung von Union und Kirche zu bekräftigen. ${ }^{20}$ Gerade um den säkularen Charakter der EU zu wahren, müsse

17 Söbbeke-Krajewski, M., a.a.O., $261 \mathrm{f}$.

$18 \mathrm{Zu}$ den Stellungnahmen der Religions- und Weltanschauungsgemeinschaften zur Konventsdebatte vgl. Schnabel, P.R., a.a.O. $163 \mathrm{ff}$.

19 CONV 601/03 vom 11.03. 2003. Vgl. zu den Beratungen im Verfassungskonvent auch die Berichte der Beobachter von CEC, COMECE und EKD in: CEC, COMECE, EKD (Hg.): The European Convention - The Evolution of a Constitution for Europe. Reports on the Plenary Sessions of the European Convention, Brüssel, 2003.

20 Follini, M., Redebeitrag gem. Protokoll der Plenarsitzung vom 27.02. 2003; CEC, COMECE, EKD (Hg.), a. a. O., $114 \mathrm{f}$. 
eine ausgleichende Regelung zwischen Staat und Religion geschaffen werden. Daher sei es erforderlich, Religion unter ihrem institutionellen Aspekt und als Teil der Zivilgesellschaft in die Verfassung aufzunehmen.

Der Verfassungskonvent befasste sich in den Plenarsitzungen vom 3./4. April 2003 (18. Sitzung) und vom 24./25. April 2003 (19. Sitzung) in Brüssel mit dem „Kirchenartikel“, der am 3. April 2003 zunächst als Art. 37 vom Konventspräsidium dem Plenum zur Debatte vorgelegt wurde. Dieser war Teil des Titels VI des Verfassungsentwurfs „Das demokratische Leben der Union“21. Auch zu diesem Zeitpunkt enthielt Art. 37 bereits einen Abs. 3. Dieser lautete ursprünglich: „Die Union pflegt in Anerkennung der Identität und des besonderen Beitrags dieser Kirchen und Gemeinschaften einen regelmäßigen Dialog mit ihnen “22. Der Kommentar des Konventspräsidiums zu Art. 37 enthält keine Substanz; er weist lapidar auf die Erklärung Nr. 11 hin und wiederholt im Wesentlichen den Wortlaut des Absatzes 3. ${ }^{23}$ Bei der Vorstellung des Präsidiumsvorschlags im Plenum erläuterte der Vizepräsident des Konvents Dehaene, dass der Dialog der europäischen Institutionen mit den in der Vorschrift genannten Organisationen klar zu unterscheiden sei von dem Dialog mit den Organisationen der Zivilgesellschaft gemäß Art. 34 Verfassungsentwurf. ${ }^{24}$ Die Ergänzung zur Endfassung „, [...] einen offenen, transparenten und regelmäßigen Dialog [...]“ geht zurück auf einen Änderungsantrag finnischer Abgeordneter. ${ }^{25}$ Diese Abgeordneten - sie standen einem „Kirchenartikel“ durchaus kritisch gegenüber begründeten ihren Antrag damit, dass der Dialog nach Art. 37 Abs. 3 Verfassungsentwurf auf denselben Prinzipien beruhen solle wie der Dialog mit der Zivilgesellschaft gemäß Art. 34 Abs. 3 Verfassungsentwurf. ${ }^{26}$ Die Ergänzung

21 CONV 650/03 vom 02.04. 2003.

22 Engl. ,, The Union shall maintain a regular dialogue with these churches and organisations, recognising their identity and their specific contribution. “; frz. „L'Union maintient un dialogue régulier avec ces églises et organisations, en reconnaissance de leur identité et leur contribution spécifique. “

$23 \mathrm{Zu}$ Art. 37: „, Comments: 1. Paragraphs 1 and 2 take over in full the text of Declaration 11 annexed to the Amsterdam Treaty, on the status of churches and non-confessional organisations. 2. Paragraph 3 specifies that the Union is to maintain a dialogue with the churches and organisations referred to in paragraphs 1 and 2 (as with the associations and civil society; see Article 34). ", CONV 650/03 vom 02.04. 2003.

24 CEC, COMECE, EKD (Hg.), a. a. O., 127.

25 Es handelte sich um die Abgeordneten Tiilikainen, Peltomäki, Kiljunen, Vanhanen und Korhonen.

26 Die Begründung im Änderungsantrag lautet: ,, We take a critical view as regards the need for a separate article in the Constitution concerning churches and non-confessional organisations. In our view, these could well be included in Article 34 paragraph 3, together with representative associations and civil society. If, however, the Convention should choose to retain the provision, the dialogue envisaged in paragraph 3 should be based on the same principles as the dialogue in Article 34 paragraph 3. “ 
beruht somit auf einer Abgleichung des Textes mit der Vorschrift zum Dialog mit der Zivilgesellschaft.

Die Debatte über den Präsidiumsvorschlag wurde auf der 19. Plenarsitzung am 24./25. April 2003 geführt. Nach der Vorstellung des Präsidiumsentwurfs wurden aus dem Plenum etwa vierzig Änderungsanträge vorgebracht, die jedoch oftmals weitgehend deckungsgleich waren und daher in Kategorien zusammen gefasst werden können. In der lebhaften Debatte äußerte sich eine große Zahl der Delegierten.

Eine Anzahl von Abgeordneten sprach sich gegen Art. I-52 Abs. 1, 2 VVE aus und verlangte dessen Streichung. Teilweise hielten sie die Vorschrift für überflüssig, da sie der Ansicht waren, dass die EU ohnehin keine Kompetenz zur Regelung des Verhältnisses Staat-Kirche in den Mitgliedstaaten habe. Manche meinten, dass Art. I-52 Abs. 1, 2 VVE redundant sei, da sein Inhalt sich bereits aus Art. 10 Abs. 1 EGRC ergebe. ${ }^{27}$ Eine Delegierte warnte vor unvorhergesehenen Konsequenzen, wenn eine unverbindliche Erklärung in verbindliches Primärrecht umgewandelt wird. ${ }^{28}$ Das im Vertrag von Amsterdam gefundene Gleichgewicht über das Statut der Kirchen solle nicht angetastet werden; dies könne aber geschehen, wenn die Erklärung Rechtsstatus erhalte. Juristische Probleme könnten auftreten, wenn Rechte für bestimmte weltanschauliche Organisationen geschaffen werden, daher sei eine präzise juristische Analyse zu diesem Punkt erforderlich. Andere Abgeordnete warnten vor den Gefahren bestimmter Organisationen oder der Religion generell. So wurde befürchtet, dass Scientology oder andere zweifelhafte Organisationen durch die Klausel in den Mitgliedstaaten begünstigt werden könnten, ${ }^{29}$ oder es wurde auf die Vielzahl von Kriegen verwiesen, die in Europa im Namen der Religion geführt worden seien. ${ }^{30}$

Demgegenüber wurde für die Aufnahme des Art. I-52 Abs. 1, 2 VVE angeführt, dass es notwendig sei, die Achtung der Union für die verschiedenen staats-kirchenrechtlichen Systeme in der EU in der Verfassung zu verankern; ${ }^{31}$ dies wurde zum Teil auch mit dem Hinweis auf Subsidiarität ${ }^{32}$ und dem Schutz der nationa- 
len Identität ${ }^{33}$ begründet. Art. I-52 Abs. 1, 2 VVE verdeutliche, dass die EU eine Wertegemeinschaft $\mathrm{se}^{34}$ und bekräftige die Relevanz der dort genannten Organisationen für die Gesellschaft. ${ }^{35}$ Die Norm sei nicht nutzlos neben Art. 10 Abs. 1 EGRC, sondern komplementär zu diesem; ein Dialog zwischen Politik und den Religionen sei eine Notwendigkeit, für die die Voraussetzungen geschaffen werden müssen; daher enthielten so gut wie alle mitgliedstaatlichen Verfassungen besondere Vorschriften betreffend Religion, Kirchen und ähnliche Organisationen. Der vorgeschlagene Artikel sei Ausdruck eines authentischen demokratischen Ansatzes der EU. ${ }^{36}$

Die Debatte wurde in Ansätzen auch um den Umfang des Art. I-52 Abs. 1, 2 VVE geführt. Einige Delegierte machten Vorschläge, Art. I-52 Abs. 1, 2 VVE noch zu ergänzen, um bestimmte Partikularinteressen einzufügen oder zu betonen. So wurde angeregt, auch die kirchlichen Schulen ausdrücklich zu erwähnen, um sie vor europarechtlichem Einflusse zu schützen. ${ }^{37}$ Manche Teilnehmer wollten die Toleranz für weltanschauliche Gemeinschaften daran binden bzw. darauf beschränken, dass diese die Werte der EU-Verfassung respektieren ${ }^{38}$ oder die Unverletzlichkeit der menschlichen Person achten, da verhindert werden müsse, dass sich diese Organisationen der Klausel bedienen, um illegale und kriminelle Aktivitäten zu rechtfertigen. ${ }^{39}$ Ein Abgeordneter regte an, die Geltung des Art. I52 Abs. 1, 2 VVE zumindest in der Auslegung auf Aktivitäten zu beschränken, die der Förderung des Glaubens dienen; die Klausel solle aber die Religionsgemeinschaften nicht vor den Auswirkungen des europäischen Arbeitsrechts schützen. ${ }^{40}$ Gerade der letzte Vorschlag würde zu einer erheblichen Einschränkung der Achtungsklausel führen und zeigt, dass es im Konvent durchaus Vorstellun-

33 Brok, E., Haenel, H., Redebeiträge gem. Protokoll der Plenarsitzung vom 24. 04. 2003.

34 Fischer, J., Redebeitrag gem. Protokoll der Plenarsitzung vom 24.04. 2003.

35 Fischer, J., Olesky, J., Redebeiträge gem. Protokoll der Plenarsitzung vom 24.04. 2003.

36 Figel, J., Redebeitrag gem. Protokoll der Plenarsitzung vom 24.04. 2003.

37 Heathcoat-Amory, D., Redebeitrag gem. Protokoll der Plenarsitzung vom 24.04. 2003.

38 Muscardini, C., Redebeitrag gem. Protokoll der Plenarsitzung vom 24.04. 2003.

39 Lequiller, P., Redebeitrag gem. Protokoll der Plenarsitzung vom 24. 04. 2003.

40 De Rossa, P., Redebeitrag gem. Protokoll der Plenarsitzung vom 24.04. 2003: „I am also concerned about Article 37 (1), which seeks not to prejudice the status under national law of churches and religious associations or communities in the Member States. I have no problem with that if all it means is that there would be no interference in their activities in relation to promoting their faith. However, in my own country the national law actually precludes them from having to comply with labour law. Recently, in the European Parliament we have dealt with a specific instance of that in the UK. We should not give a blanket exclusion for religious orders or churches enabling them to get out of European labour law. “ 
gen gab, die keine reine Schutzklausel für nationale staatskirchenrechtliche Systeme favorisierten, sondern die Achtung dieser Systeme ausdrücklich unter europarechtliche Bedingungen stellen wollten. Unabhängig von der Frage, inwieweit nationale Systeme trotz des Art. I-52 VVE vom Unionsrecht betroffen werden, haben sich die Vorschläge für eine „konditionale Achtungsklausel“ im Konvent nicht durchgesetzt.

Die Dialogklausel des Art. I-52 Abs. 3 VVE war oft Gegenstand gesonderter Argumentation. Die Kritiker verwiesen in Änderungsanträgen und Redebeiträgen darauf, dass eine besondere Erwähnung des Dialogs mit den Religionsgesellschaften überflüssig sei, weil diese Teil der Zivilgesellschaft seien und somit schon der Vorschrift über den allgemeinen Dialog mit deren Organisationen unterfiele (Art. I-46 Abs. 2 VVE), außerdem bedeute eine gesonderte Erwähnung eine ungerechtfertigte Privilegierung. ${ }^{41}$ Die Befürworter betonten hingegen drei Argumente für eine besondere Erwähnung des Dialoges: den besonderen Beitrag der religiösen Organisationen zur europäischen Einigung (wobei ungesagt blieb, worin dieser bestehe $)^{42}$, den besonderen Charakter des Dialogs ${ }^{43}$, und das Phänomen, dass die Gesetzgebung der Union in vielen Bereichen die Belange religiöser Gemeinschaften berührt, auch wenn sie sie nicht primär zum Regelungsziel nimmt, sondern inzidenter betrifft. Es wurde argumentiert, dass die Akzeptanz der EU-Rechtsakte und Politiken seitens dieser Gemeinschaften bedeutend steigen könne, wenn sie in einem strukturierten Dialog mit diesen Organisationen entstünden. ${ }^{44}$

Diese Untersuchung der Konventsmaterialien zeigt, dass eine ausführliche Befassung des Konvents mit dem Entwurf des Art. I-52 VVE stattgefunden hat. Dabei standen allerdings politische Fragen wie die nach der Union als Wertegemeinschaft oder der Notwendigkeit einer gesonderten Erwähnung des Dialogs im Vordergrund. Juristische Fragen, die sich aus der Übernahme der Erklärung Nr. 11 in das Primärrecht ergeben, wurden demgegenüber nur vereinzelt gestreift. ${ }^{45}$

41 Demiralp, O., Fayot, B., Borell Fontelles, J., McAvan, L., de Rossa, P., Redebeiträge gem. Protokoll der Plenarsitzung vom 24.04. 2003.

42 Teufel, E., Wittbrodt, E., Redebeiträge gem. Protokoll der Plenarsitzung vom 24. 04. 2003.

43 Brok, E., Redebeitrag gem. Protokoll der Plenarsitzung vom 24. 04. 2003.

44 Serracino-Inglott, P., Redebeitrag gem. Protokoll der Plenarsitzung vom 24.04. 2003: „,...] there are many areas where European laws have touched on religious issues in ways which do not belong strictly and exclusively to the competence of the Member States. The structured dialogue would make the policies of the Union much more generally acceptable. "

45 Andreani, P., Redebeitrag gem. Protokoll der Plenarsitzung vom 24. 04. 2003. 


\section{Die Achtungsklausel des Art. 17 Abs. 1 und 2 AEUV}

Gemäß Art. 17 Abs. 1 AEUV achtet die Union den Status, den Kirchen und religiöse Vereinigungen oder Gemeinschaften in den Mitgliedstaaten nach deren Rechtsvorschriften genießen, und beeinträchtigt ihn nicht. Art. 17 Abs. 2 AEUV erstreckt diese Achtung in gleicher Weise auf den Status von weltanschaulichen Gemeinschaften.

\section{Tatbestandsmerkmale}

\section{a) Union}

Die Vorschrift verpflichtet die Europäische Union. Damit bezieht sich die Norm auf das gesamte staatsähnliche Gebilde, das gemäß Art. 1 EUV geschaffen wird. Art. 17 AEUV gilt somit für die Union als ganzes; er hat Bedeutung für sämtliche Organe und sämtliche Handlungen der Union und ist nicht auf bestimmte Institutionen, Politikfelder oder Tätigkeitsbereiche beschränkt. Jedes Organ hat bei jeder für die Union vorgenommenen Rechtshandlung die Vorschrift zu beachten. Sie gilt für die Ausübung der ausschließlichen Zuständigkeiten ebenso wie für die Ausübung der geteilten Zuständigkeiten (Art. $2 \mathrm{ff}$. AEUV). Sie gilt gleichermaßen für die Unterstützungs-, Koordinierungs- und Ergänzungsmaßnahmen gemäß Art. 6 AEUV. Auf letzteres hinzuweisen ist von besonderer Bedeutung, da diese Maßnahmen oftmals als Einfallstor angesehen werden, durch das die Union außerhalb ihrer Kompetenzen in das Recht der Mitgliedstaaten hineinzuwirken versucht. $\mathrm{Zu}$ den Bereichen, in denen die Union nach Art. 6c unterstützend, koordinierend und ergänzend wirken darf, gehört auch die Kultur. Diese weist oftmals eine besondere Nähe zum religionsrechtlichen Bereich auf. Daher kann die Achtungsklausel des Art. 17 Abs. 1, 2 AEUV hier eine spezifische Relevanz entwickeln. In Art. 17 Abs. 1, 2 AEUV ähnelt in der Formulierung im Übrigen Art. 8 EUV, nach dem die EU den Grundsatz der demokratischen Gleichheit aller Bürger der Union achtet. Die Parallelität mit diesem hochwertigen Rechtsgut zeigt die Bedeutung, die der religionsrechtlichen Achtungsklausel im VVE zukommt. 


\section{b) Kirchen, religiöse Vereinigungen und Gemeinschaften, weltanschauliche Gemeinschaften in den Mitgliedstaaten}

Diese Begriffe beschreiben die Organisationen und Personenmehrheiten, die Nutznießer der Achtungsklausel sind. Die lange Reihung der Begriffe zeugt von der Schwierigkeit, einen umfassenden Oberbegriff für die Erscheinungsformen organisierten religiösen Lebens innerhalb der Europäischen Union zu finden. Sie ersetzt den fehlenden Oberbegriff und ist daher nicht als abschließende Aufzählung zu verstehen. Durch die Bandbreite der Begriffe von Kirchen bis zu Gemeinschaften wird ausgesagt, dass der Schutz der Achtungsklausel allen korporativen und kollektiven Organisationsformen religiösen Lebens zuerkannt wird.

Aus mehreren Gründen kann es sich bei den aufgezählten Termini nicht um autonome Begriffe des Unionsrechts handeln. ${ }^{46}$ Zum einen ist die Union nicht befugt, die Begrifflichkeiten des Staatskirchenrechts zu regeln, da ihr für ein institutionelles Staatskirchenrecht keine Kompetenzen zuerkannt sind. Die Achtungsklausel des Art. 17 Abs. 1, 2 AEUV selbst gewährt gerade keine Kompetenz dafür, sondern soll das Entstehen eines Unions-Kirchenrechts, das die nationalen Staatskirchenrechte verdrängt, verhindern. Nach dem Prinzip der begrenzten Einzelermächtigung (Art. 5 Abs. 1 EUV neu) kann nur die ausdrückliche Übertragung einer entsprechenden Kompetenz die autonome unionsrechtliche Ausfüllung der Begriffe mit staatskirchenrechtlichem Inhalt ermöglichen; eine solche Übertragung existiert jedoch nicht. Außerdem spricht die Klarstellung, dass die religiösen Gruppierungen ihren Status nach den mitgliedstaatlichen Rechtsvorschriften genießen, gegen die Annahme autonomer unionsrechtlicher Begriffe. Eine solche Bezugnahme auf nationale Normen macht nur Sinn, wenn das nationale Recht gerade nicht durch unionsrechtliche Begriffsdefinitionen überlagert und in der Anwendung verdrängt wird. Die in Art. 17 Abs. 1, 2 AEUV gebrauchten Begriffe enthalten demnach keine autonome unionsrechtliche Bedeutung, sondern beziehen ihren Gehalt aus den nationalen staatskirchenrechtlichen Regelungen. Die Achtungsklausel schützt damit alle Regelungsobjekte, die

46 A. A. Vachek, M., a. a. O., 142 ff. Vachek geht schon bei der Erklärung Nr. 11 von, ,gemeinschaftsrechtlichen Begriffsprägungen“" aus, die eine autonome, gemeinschaftsrechtliche Auslegung ermöglichen. Diese Auffassung ist möglicherweise durch das Anliegen Vacheks motiviert, den Schutz von kleinen Religionsgemeinschaften und Sekten, den er in den Mitgliedstaaten als defizitär wahrnimmt, durch eine Religionsrechtsentwicklung auf europäischer Ebene zu stärken (vgl. ebd., 8). Eine solche Entwicklung wäre allerdings - unabhängig von einem Urteil über ihre Wünschbarkeit - eine Beeinflussung des nationalen Staatskirchenrechts, die gerade nicht vom Zweck der Achtungsklausel erfasst ist. Im Ergebnis wertet Vachek die Erklärung dann auch lediglich als Versuch einer Festschreibung des nationalen Staatskirchenrechts, vor deren Gefahren er allerdings warnt (ebd., 152). 
nach nationalen Rechtsvorschriften unter die gebrauchten „Begriffshülsen“ subsumiert werden können. Sie erfasst folglich die Vielfalt der mitgliedstaatlichen staatskirchenrechtlichen Organisationsformen. Im Ergebnis bedeutet dies, dass der Rechtskreis einer Personenmehrheit, die nach dem Recht eines Mitgliedstaates als Religionsgemeinschaft eingestuft wird und der demnach auf nationaler Ebene bestimmte Rechte zu Teil werden, in den Anwendungsbereich des Art. 17 Abs. 1, 2 AEUV fällt; wird eine gleichartige Personenmehrheit in einem anderen Mitgliedstaat nicht als religiöse Vereinigung o. ä. anerkannt, so bleibt Art. 17 Abs. 1, 2 AEUV insoweit für diese bedeutungslos.

Dem gegenüber sei angemerkt, dass die Berechtigten aus dem korporativen Grundrecht der Religionsfreiheit des Art. 10 Abs. 1 EGRC autonom unionsrechtlich bestimmt werden. ${ }^{47}$ Dies bedeutet keinen Widerspruch zwischen Art. 10 Abs. 1 EGRC und Art. 17 Abs. 1, 2 AEUV. In Art. 10 Abs. 1 EGRC wird ein eigenes unionsrechtliches Grundrecht definiert. Es geht dort somit eine genuin unionsrechtliche Materie. In Art. 17 AEUV hingegen geht es um die unionsrechtliche Rücksichtnahme auf Rechtspositionen, die sich aus dem nationalen Recht der Mitgliedstaaten ergeben.

\section{c) Status}

Gegenstand der Achtung, die Art. 17 AEUV gewährt, ist der Status der Religionsgemeinschaften, den die mitgliedstaatlichen Rechtsvorschriften diesen gewähren. Eine Legaldefinition des Merkmals „Status“ fehlt. ${ }^{48}$ Die Wahl des Begriffs „Status“ legt nahe, dass keine pauschale Bereichsausnahme für alle religionsrechtlich relevanten Regelungen der Mitgliedstaaten beabsichtigt ist; ${ }^{49}$ wäre dies bezweckt worden, wäre es naheliegender gewesen, an Stelle von „Status“ den Ausdruck „die Rechte [der Religionsgemeinschaften]“ zu benutzen, oder zu erklären, dass nationale religionsrechtliche Regelungen vom EU-Recht unberührt bleiben. Der Begriff „Status“ deutet also auf einen demgegenüber reduzierten Gehalt der Norm hin. Sinn und Zweck des Art. 17 AEUV wie auch der vorhergehenden Erklärung Nr. 11 ist es, die institutionelle Struktur des Verhältnisses zwischen Religionsgemeinschaften und Staat in den Mitgliedstaaten

48 Vgl. Grzeszick, B., a. a. O., 294; de Wall, H.: Europäisches Staatskirchenrecht, in: Zeitschrift für evangelisches Kirchenrecht, 45/1 (2000), 157-172, hier 158; Muckel, S., a. a. O., $198 \mathrm{f}$.

49 Muckel, S., a. a.O., 199. 
vor verändernden Eingriffen des EU-Rechts zu sichern. ${ }^{50}$ Beispiele für die institutionelle Struktur des Staat-Kirche-Verhältnis sind etwa die Arrangements zwischen Kirchen und dem Staat zur Erteilung kirchlichen Religionsunterrichtes in staatlichen Schulen; Teilhaberechte in Gremien, die über die Programmgestaltung des öffentlichen Rundfunks mitbestimmen; Teilhaberechte für kirchliche Amtsträger an Legislativorganen des Staates (beispielsweise Parlamentssitze kraft kirchlichen Amtes im britischen Oberhaus) sowie die staatliche Beteiligung an den nationalen Modellen der Kirchenfinanzierung. Dementsprechend ergibt sich der „Status“ aus dem Bestand an rechtlichen Normen, die das Staatskirchenrecht der Mitgliedstaaten jeweils bereitstellt, um diese institutionelle Struktur zu gestalten. Damit wählt Art. 17 AEUV einen institutionellen Ansatz ${ }^{51}$, der dem Ansatz der mitgliedstaatlichen staatskirchenrechtlichen Systeme eher entspricht als dem typischerweise dynamischen, funktionalen Regelungsansatz des Unionsrechts. Auch dies verdeutlicht, dass es hier um den Schutz vorgefundener nationaler Strukturen geht. Indem die Achtung der nationalen staatskirchenrechtlichen Systeme gewährleistet wird, ist ein direkter Eingriff seitens der EU in die institutionellen Strukturen des nationalen Staatskirchenrechts ausgeschlossen. Versperrt ist damit der Weg zu einem institutionellen Unions-Kirchenrecht, das im Wege des Anwendungsvorrangs nationalem Staatskirchenrecht vorginge.

Fraglich ist aber, ob und wie weit der Schutz des Art. 17 Abs. 1, 2 AEUV über die institutionelle Struktur des nationalen Staat-Kirche-Gefüges hinaus sich auf die Gesamtheit der Rechte bezieht, die der Status einer Religionsgemeinschaft in einem Mitgliedstaat mit sich bringt. In den Mitgliedstaaten gehen die an den Status geknüpften Rechte oft über die institutionelle Gestaltung des StaatKirche-Verhältnisses hinaus. Manche nationalen staatskirchenrechtlichen Systeme leiten aus dem besonderen institutionellen Status von Religionsgemeinschaften besondere funktionale Rechte für diese her, beispielsweise besondere arbeitsund wirtschaftsrechtliche Regeln. Fasste man das gesamte Staatskirchenrecht eines Mitgliedstaates schlechthin unter den Begriff Status, stünde man vor einem Dilemma. Im Ergebnis käme man dann über Art. 17 Abs. 1, 2 AEUV zu einer je nach Mitgliedstaat sehr weitreichenden Bereichsausnahme für die gesamten rechtlichen Beziehungen von Religionsgemeinschaften. ${ }^{52}$ Diese These ist jedoch

50 Zum telos der Struktursicherung vgl. Mückl, S., a. a. O., 455.

51 Walter, C., a. a. O., $418 \mathrm{f}$.

52 Weitgehend Robbers, G.: Religion in Europa. Die religionsgeschichtliche Bedeutung der neuen Verfassung für die Europäische Union, in: Informationes Theologiae Europae, 12 (2003), 9-22, hier $11 \mathrm{f}$., der Kirchen auflistet, wenn sie als Arbeitgeber Krankenhäuser, Kindergärten oder Banken betreiben, und 
mit Hinweis auf den Wortlaut bereits zurückgewiesen worden. Andererseits erscheint es nicht überzeugend, den von Art. 17 Abs. 1, 2 AEUV gewährten Schutz nur auf das institutionelle staatskirchenrechtliche Gefüge der Mitgliedstaaten zu beschränken. Ein solcher Schutz ist zwar symbolisch wertvoll, inhaltlich jedoch mit Blick auf die ohnehin fehlende Kompetenz der Union zur Schaffung institutioneller staatskirchenrechtlicher Regeln redundant. ${ }^{53}$ Doch trotz des Fehlens einer staatskirchenrechtlichen Kompetenz konstatieren gerade die etablierten Religionsgemeinschaften einen europarechtlich induzierten Veränderungsdruck auch im institutionellen Staat-Kirche-Gefüge auf der nationalen Ebene. Dies ist darauf zurückzuführen, dass die inzidente Betroffenheit von Religionsgemeinschaften in den Mitgliedstaaten durch Rechtsakte der EU mittelbare Veränderungen auf die nationalen staatskirchenrechtlichen Strukturen verursacht. ${ }^{54}$ Solch ein mittelbarer Effekt wurde beispielsweise an Hand des Vorschlages einer Datenschutzrichtlinie sichtbar, die das deutsche Verfahren des Kirchensteuereinzugs in Frage zu stellen schien. Auswirkungen dieser Art waren es, die die Religionsgemeinschaften dazu bewogen, auf die Erklärung Nr. 11 zu drängen, in der der Begriff „Status“ gewählt wurde. Dieser Hintergrund legt nahe, dass mit der Norm bezweckt wurde, über das institutionelle Gefüge hinaus den Veränderungsdruck auf die etablierten staatskirchenrechtlichen Strukturen aufzufangen. Aus dieser ratio legis ist zu schließen, dass die Norm mit dem Begriff „Status“ eine Struktur sichernde Wirkung erzielen soll. ${ }^{55}$ Daraus ist abzuleiten, dass der in Art. 17 Abs. 1, 2 AEUV enthaltene Schutz des „Status“ neben dem institutionellen staatskirchenrechtlichen Gefüge die strukturgebenden nationalen religionsrechtlichen Normen umfassen soll. Diese bestehen in denjenigen Regelungen, die für das nationale Religionsrecht wesentlich sind, weil sie einen Identität begründenden, charakteristischen, grundsätzlichen oder das nationale System etablierenden Inhalt haben. Darunter können beispielsweise Teilhaberechte in staatlichen Institutionen, Regelungen der Finanzierung der Religionsgemeinschaften und besondere arbeitsrechtliche Regeln fallen. Andererseits werden so nicht alle religionsrechtlichen Regelungen der Mitgliedstaaten erfasst. Somit bietet Art. 17 Abs. 1, 2 AEUV keinen umfassenden Schutz vor einer Inzi-

Ordensgemeinschaften, die ihren Unterhalt aus Landwirtschaft oder Gärtnereien beziehen. Soweit für diese Bereiche besondere religionsrechtliche Bestimmungen in den Mitgliedstaaten bestehen, müsse die EU die daraus resultierende Stellung der Religionsgemeinschaften achten und dürfe sie nicht beeinträchtigen; zurückhaltender Muckel, S., a. a. O., 199.

53 Walter, C., a. a. O., 418; Mückl, S., a. a. O., 455 f.; Rüfner, W., a.a. O., 770.

54 Rüfner, $W$., a. a.O., 767.

55 Walter, C., a. a. O., 412, 416; Mückl, S., a.a.O., 455 f.; Naumann, K., a. a. O., 202. 
dent-Betroffenheit der Religionsgemeinschaften durch das Unionsrecht, wie sie sich in vielfältiger Weise durch sektorale oder Querschnitts-Gesetzgebung oder andere Rechtsakte der Union ergibt, sondern schützt das institutionelle staatskirchenrechtliche Gefüge plus die wesentlichen, tragenden, strukturgebenden Regelungen des nationalen Religionsrechts. Das bedeutet, dass Art. 17 Abs. 1, 2 AEUV einen beschränkten Schutzansatz verfolgt, der nicht zu einer Bereichsausnahme für die Religionsgemeinschaften und ihre rechtlichen Beziehungen ausgedehnt werden kann.

\section{d) Achten und nicht-beeinträchtigen}

Art. 17 Abs. 1, 2 AEUV verpflichtet die Union, den so definierten Status zu achten und nicht zu beeinträchtigen. Fraglich ist, welche Bedeutung die beiden Verben konkret enthalten. Das Wort „achten“ findet sich oft in Verfassungen (etwa Art. 1 GG), ohne definiert zu sein. Sein Bedeutungsgehalt ist so flexibel, dass die Schutzintensität erst im Zusammenspiel mit dem Gewicht des zu achtenden Rechtsgutes entsteht (eine hohe Schutzintensität beispielsweise bei der Achtung der Menschenwürde). Damit wird klar, dass ,achten“ die Bildung eines Werturteils verlangt, auf dessen Grundlage dem zu schützenden Rechtsgut dann der ihm gebührende Platz zugewiesen wird. Das Gebot des Achtens enthält damit auch eine Verhältnismäßigkeitskomponente, denn es setzt einen Abwägungsvorgang voraus, in dessen Rahmen es einen Platz findet. ${ }^{56}$ „Nicht beeinträchtigen“ meint, dass keine verschlechternden Rechtsakte und Handlungen vorgenommen werden, die den Umfang des Status verringern. Die (Nicht-)Beeinträchtigung ist dabei in dem Sinne statisch, dass sie durch einen Vergleich der Rechtslage ohne den fraglichen EU-Rechtsakt mit der hypothetischen Rechtslage mit dem EURechtsakt festgestellt werden kann; sie hängt im Gegensatz zum Achten nicht von einer Abwägung ab. ${ }^{57}$ Fällt die Bilanz der Statusrechte der Religionsgemeinschaften unter dem EU-Rechtsakt im Vergleich zu ihren Rechtspositionen ohne EU-Rechtsakt negativ aus (d.h. eine Religionsgemeinschaft hat unter dem hypothetischen EU-Rechtsakt weniger oder weniger umfangreiche Statusrechte), liegt eine Beeinträchtigung vor. Dabei ist noch einmal darauf hinzuweisen, dass sich das Verb „beeinträchtigen“ auf den oben definierten Status bezieht und dessen

56 Im Gegensatz zum Begriff ,Achten“ wäre etwa die Wortwahl ,garantiert“ in dem Sinne stärker gewesen, als dass dabei das Ergebnis der Wertbeimessung prädeterminiert wäre in dem Sinne, dass der Status in jedem Falle zu wahren sei, egal, welcher Wert dem konkurrierenden Ziele zukommt.

57 Zur Bedeutung dieses Unterschiedes vgl. Söbbeke-Krajewski, M., a. a. O., $272 \mathrm{f}$. 
Verschlechterung verhindern soll, aber nicht das gesamte nationale Religionsrecht im Sinne einer Bereichsausnahme von Eingriffen freistellt.

Vor diesem Hintergrund ergeben sich für die Achtungsklausel drei Kategorien unterschiedlicher praktischer Bedeutung. In Bezug auf das institutionelle Staatskirchenrecht der Mitgliedstaaten bekräftigt die Achtungsklausel, dass die Union nicht eingreifen darf. Dies ergibt sich allerdings nicht erst aus Art. 17 Abs. 1, 2 AEUV, sondern daraus, dass der Union keine Kompetenz zugewiesen ist, primär staatskirchenrechtliche Fragen zu regeln. ${ }^{58}$ Insoweit ist die Achtungsklausel also deklaratorisch. In Bezug auf inzidente Betroffenheit der Religionsgemeinschaften durch Rechtsakte der EU, für die diese die Kompetenz besitzt, bietet Art. 17 Abs. 1, 2 AEUV keinen Schutz im Sinne einer Bereichsausnahme, da lediglich der Status der Religionsgemeinschaften geschützt wird, aber nicht ihre gesamten Rechte von der Geltung des EU-Rechts ausgenommen werden. Praktische Bedeutung entwickelt Art. 17 Abs. 1, 2 AEUV aber dort, wo die inzidente Betroffenheit auf nationalrechtlich gewährte institutionelle Rechtspositionen oder Struktur prägende Regeln des nationalen Religionsrechts (also auf den „Status“ im oben erläuterten Sinne) übergreift. Ist die Betroffenheit von der Art, dass institutionelle Positionen oder Struktur prägende Regelungen durch EU-Recht beeinträchtigt werden, so lässt sich aus Art. 17 Abs. 1, 2 AEUV die Pflicht der Union ableiten, ihren Rechtsakt so zu fassen, dass eine Verringerung der Statusrechte der Religionsgemeinschaft vermieden wird. Nur dann wird nämlich deren statusrechtliche Position geachtet und nicht beeinträchtigt. Dies kann etwa durch Ausnahmeregelungen im EU-Rechtsakt gestaltet werden. Ein Beispiel dafür ist die Datenschutzrichtlinie. ${ }^{59}$ Diese stammt zwar aus der Zeit vor der Geltung des Art. 17 Abs. 1, 2 AEUV und sogar vor der Erklärung Nr. 11 zum Vertrag von Amsterdam, zeigt aber modellhaft, wie sich der Schutz, den Art. 17 Abs. 1, 2 AEUV bietet, auswirkt. Die Datenschutzrichtlinie ist ein allgemeiner Rechtsakt der EU. Eine frühe Entwurfsfassung hätte - wäre sie in Kraft getreten - das deutsche System des Kirchensteuereinzugs in Frage gestellt. Der staatliche Kirchensteuereinzug dürfte eine Struktur prägende Rechtsposition bestimmter Religionsgemeinschaften im deutschen Staatskirchenrecht darstellen. Damit wäre im Falle der ursprünglich geplanten Datenschutzrichtlinie auf eine wesentliche Rechtsposition der Religionsgemeinschaften übergegriffen und eine strukturprägende Re-

58 Mückl, S., a. a.O., 455 f.

59 Art. 8 Richtlinie 1995/46/EG des Europäischen Parlaments und des Rates vom 24.10.1995 zum Schutze natürlicher Personen bei der Verarbeitung personenbezogener Daten und zum freien Datenverkehr, ABIEU Nr. L 281/31 vom 23.11. 1995; vgl. auch Mückl, S., a. a. O., 533 ff. 
gelung des deutschen Staatskirchenrechts verändert worden. Art. 17 Abs. 1, 2 AEUV hätte in dieser Situation die EU verpflichtet, eine Ausnahmeregelung in das Richtlinienvorhaben aufzunehmen, die diese Art der Betroffenheit vermeidet.

\section{Subjektiv-rechtlicher Gehalt des Art. 17 Abs. 1, 2 AEUV}

Fraglich ist, ob die Vorschrift lediglich objektiv-rechtlichen Gehalt hat, oder ob aus ihr auch subjektiv-öffentliche Rechte herzuleiten sind. Fasst man die Norm als rein objektiv-rechtlich auf, so enthält sie einen Handlungsauftrag an die Union mit dem Inhalt, bei allen ihren Handlungen, darunter insbesondere bei ihrer gesetzgeberischen Tätigkeit, den Status der Religionsgemeinschaften zu wahren. Leitet man jedoch subjektiv-rechtlichen Gehalt aus ihr her, so versetzt man dadurch die aus der Norm Berechtigten in die Lage, die Gewährleistung gerichtlich einzuklagen und durchzusetzen. Art. 17 Abs. 1, 2 AEUV würde dadurch justitiabel.

\section{a) Subjektive Rechte im Unionsrecht}

Der EuGH hat bereits in der Rechtssache van Gend \& $\operatorname{Loos}^{60}$ festgestellt, dass Normen des Primärrechts unmittelbare Wirkung entfalten, also Private unmittelbar verpflichten und berechtigen können, auch wenn sie nicht mitgliedstaatlich umgesetzt worden sind. Auch Art. 17 Abs. 1, 2 AEUV könnte also, wenn bestimmte Bedingungen erfüllt sind, den vorgesehenen Rechtssubjekten individuelle Rechtsmacht verleihen. Festzuhalten ist, dass es sich bei Art. 17 Abs. 1, 2 AEUV um eine Norm handelt, die - wie die Unionsgrundrechte - nur die Union bindet und keiner nationalen Umsetzung bedarf.

Diese Frage nach dem subjektiv-rechtlichen Gehalt einer Norm löst der EuGH nach einer Methode, die der aus dem deutschen Recht bekannten Schutznormtheorie sehr ähnelt. ${ }^{61}$ Er prüft, ob die betreffende Rechtsnorm die Verleihung von Individualrechten bezweckt, ${ }^{62}$ und ob der Petent im Schutzbereich der Norm

60 EuGH, Urteil v. 05.02.1963, Rs. 26/62, Slg. 1963, 1 ff. (van Gend \& Loos).

61 Triantafyllou, D.: Zur Europäisierung des subjektiven öffentlichen Rechts, in: Die öffentliche Verwaltung, 50/5 (1997), 192-200, hier 195 f.; EuGH, Urteil v. 20.09. 1988, Rs. 31/87, Slg. 1988, 4635 ff., hier Rn. 42 ff. (Beentjes); EuGH, Urteil v. 11.08.1995, Rs. C-433/93, Slg. 1995, I-2303 ff., hier Rn. 19 (Kommission ./. Deutschland).

62 Vgl. etwa EuGH, Urteil v. 19.11. 1991, verb. Rs. C-6/90 und C-9/90, Slg. 1991, I-5357 ff., hier Rn. 22, 40 (Francovich). 
steht. ${ }^{63}$ Diese beiden Voraussetzungen sind also auch für Art. 17 Abs. 1, 2 AEUV zu prüfen.

\section{b) Untersuchung des Art. 17 Abs. 1, 2 AEUV auf subjektiv-rechtlichen Gehalt}

Ob Art. 17 Abs. 1, 2 AEUV subjektive Rechte der Religionsgemeinschaften zu schützen bezweckt, ist an Hand einer Auslegung der Norm selbst zu ermitteln. ${ }^{64}$ Hierzu sind Wortlaut, Entstehungsgeschichte, Systematik und Regelungszweck zu untersuchen. ${ }^{65}$

Fraglich ist, ob der Wortlaut für oder gegen einen Individualschutz spricht. Die Formulierung ,achtet“ und „beeinträchtigt nicht“ ist insofern nicht eindeutig. Einerseits ist sie nicht so klar gefasst wie z. B. Art. 15a AEUV, der formuliert „Jeder Mensch hat das Recht $[\ldots]$ “. . So hätte Art. 17 Abs. 1, 2 AEUV auch gefasst werden können als „Religionsgemeinschaften haben das Recht auf Achtung und Nicht-Beeinträchtigung“, um den subjektiven Gehalt herauszustellen. Aber der tatsächlich gewählte Wortlaut schließt nicht aus, dass Individualrechtsschutz möglich ist.

Recht von Verfassungsrang bedarf oftmals der Konkretisierung durch einfaches Recht, aus dem dann subjektive Rechte hergeleitet werden. Aber für den Fall, dass eine Norm von Verfassungsrang nicht sekundärrechtlich umgesetzt wird oder gar nicht zur Umsetzung vorgesehen ist, kann auch aus Verfassungsrecht und EU-Primärrecht direkt ein subjektives Recht hergeleitet werden ${ }^{66}$ - so etwa aus den Grundfreiheiten und Grundrechten. ${ }^{67}$ Daher spricht auch der verfassungsrechtliche Rang von Art. 17 Abs. 1, 2 AEUV nicht gegen einen Individualrechtsschutz.

Nach Sinn und Zweck soll Art. 17 Abs. 1, 2 AEUV die gesamte Tätigkeit der Union daran hindern, unzulässigerweise in den vom nationalen Staatskirchenrecht gewährten Status einzugreifen. Insbesondere zielt die Norm aber auf den gesetzgeberischen Prozess der Union ab, dessen Ergebnisse die eigentliche Gefahr für den Status darstellen können. Im legislativen Prozess verfügt der Gesetzgeber über ein Initiativrecht und eine Einschätzungs- und Gestaltungspräro-

63 Vgl. etwa EuGH, Urteil vom 05.03.1996, verb. Rs. C-46/93 und C-48/93, Slg. 1996, I-1029ff., hier Rn. $50 \mathrm{ff}$. (Brasserie du Pêcheur).

64 Ehlers, D.: § 7, Rn. 7, in: ders. (Hg.): Europäische Grundrechte und Grundfreiheiten, Berlin, 2003.

65 Triantafyllou, D., a. a. O., $196 \mathrm{f}$.

66 Ebd., 197.

67 Ehlers, D., a.a. O., § 7, Rn. 7 f., § 13, Rn. 25 f. 
gative. Zudem ist der Gesetzgebungsprozess nicht aus einer Hand planbar, da jedes beteiligte Organ seine bestimmten Rechte hat und politische Faktoren erhebliche Rollen spielen. Ob sich Kommission, Ministerrat und Parlament für eine bestimmte Ausnahmeregelung in einem Sekundärrechtsakt zu Gunsten betroffener Religionsgemeinschaften aussprechen, hängt von der Willensbildung dieser Organe ab, die nicht einklagbar ist. Von daher könnte es schwierig sein, ein subjektives Recht in diesen Prozess einzuführen. Eine Lösung wie oben angedeutet, in Gestalt einer Ausnahmeregelung für den Fall einer drohenden Statusverletzung, ist nicht im Gesetzgebungsprozess justitiabel. Dies könnte dafür sprechen, dass Art. 17 Abs. 1, 2 AEUV nur eine Handlungsmaxime für die am Gesetzgebungsverfahren beteiligten Organe ist, dem aber kein subjektivöffentliches Recht entspricht.

Allerdings ist auch der Gesetzgeber an Recht und Gesetz gebunden. Grenzen seiner Willensbildung werden vor allem durch Kompetenzregeln und Grundrechte gesetzt. In ähnlicher Weise kann auch Art. 17 Abs. 1, 2 AEUV wirken. Bei einer Inzident-Betroffenheit des national-staatskirchenrechtlich gewährten Status einer Religionsgemeinschaft setzt Art. 17 Abs. 1, 2 AEUV dem Gesetzgeber eine materielle Grenze. Zwar enthält die Norm keine Kompetenzregelung, denn die Union besitzt ohnehin keine Kompetenz für Staatskirchenrecht, so dass Art. 17 Abs. 1, 2 AEUV diese auch nicht begrenzen kann. Vielmehr enthält die Vorschrift eine Regel, wie eine der EU zustehende Kompetenz auszuüben ist, wenn damit ein Konflikt mit dem Status einer Religionsgemeinschaft verbunden ist. Diese Situation ist vergleichbar mit derjenigen, in der ein Staat eine Kompetenz so ausübt, dass er in Grundrechte seiner Bürger eingreift. Somit ist Art. 17 Abs. 1, 2 AEUV ein grundrechtsähnliches Recht. Durch diese Einordnung wird deutlich, dass von der Systematik her nichts dagegen spricht, ihm einen subjektivrechtlichen Gehalt zu entnehmen, auch wenn die Norm primär auf das Gesetzgebungsverfahren wirken soll. Der subjektiv-rechtliche Gehalt der grundrechtsähnlichen Norm knüpft am Ergebnis des Gesetzgebungsverfahrens an (während der objektivrechtliche Gehalt sich als Handlungsauftrag bereits im Gesetzgebungsverfahren an die beteiligten Organe richtet) und wirkt dann wie die Abwehrfunktion eines Grundrechts.

Spricht also nach der bisherigen Auslegung nichts dagegen, Art. 17 Abs. 1, 2 AEUV einen subjektivrechtlichen Gehalt zu entnehmen, so bleibt zu fragen, ob die Vorschrift die positiven Voraussetzungen dafür erfüllt. Um in subjektivrecht- 
licher Hinsicht unmittelbar anwendbar zu sein, muss eine Norm hinreichend klar, vollständig und unbedingt gefasst sein. ${ }^{68}$ Hinreichend bestimmt und vollständig ist eine Norm, wenn ihr Adressat, Berechtigter und der Regelungsinhalt entnommen werden können. Nach der oben vorgenommenen Untersuchung der Tatbestandsmerkmale ist dies bei Art. 17 Abs. 1, 2 AEUV möglich. Dagegen spricht auch nicht, dass die Berechtigten und der Status auf Grund der jeweiligen nationalen Regelungen ermittelt werden müssen, denn eine Norm ist auch hinreichend klar und vollständig, wenn sie einen Verweis enthält. Der jeweilige nationale Hintergrund kann jeweils als Vorfrage zur Prüfung des Art. 17 Abs. 1, 2 AEUV ermittelt werden. Eines weiteren Umsetzungsaktes bedarf Art. 17 Abs. 1, 2 AEUV deswegen nicht. Fraglich ist aber, ob Art. 17 Abs. 1, 2 AEUV unbedingt gefasst ist. Dies ist problematisch, soweit die Norm als Regelungsinhalt das „Achten" enthält. Achten meint, wie oben dargelegt, einen Vorgang des Abwägens und Wertens, in dem der Status in eine Gesamtbetrachtung einzubeziehen ist, aber nicht um jeden Preis unberührt gelassen werden muss. Veränderungen und Einwirkungen auf den Status sind auch bei dessen Achtung nicht ausgeschlossen. In Bezug auf dieses Tatbestandsmerkmal könnte höchstens geltend gemacht werden, dass ein Abwägungsvorgang überhaupt nicht stattgefunden hat oder der Wert des Status in erheblicher Weise missachtet oder verkannt wurde. Grundsätzlich ist aber die Stellung des Status im Abwägungsvorgang des „Achtens" bedingt durch den Wert des konkurrierenden Abwägungsgutes und daher nicht unbedingt. Somit ist die Bewahrung des Status auf Grund des Gebotes des Achtens nicht subjektiv-rechtlich einklagbar. Anders allerdings verhält es sich mit dem Tatbestandsmerkmal des Nicht-Beeinträchtigens. Die Nicht-Beeinträchtigung hängt im konkreten Falle nicht von der Bewertung eines konkurrierenden Rechtsgutes ab. Sie ist daher unbedingt und feststellbar an Hand einer Überprüfung der nationalen staatskirchenrechtlichen Vorschriften vor und nach dem Erlass des EU-Rechtsaktes. Damit erfüllt Art. 17 Abs. 1, 2 AEUV die Voraussetzungen einer hinreichend bestimmten, vollständigen und unbedingten Norm insoweit, als es um das Tatbestandsmerkmal des Nicht-Beeinträchtigen geht.

Daher ist festzuhalten, dass das Tatbestandsmerkmal des Achtens lediglich objektivrechtlichen Charakter aufweist und im Gesetzgebungsverfahren der EU als

68 EuGH, Urteil v. 05.02.1963, Rs. 26/62, Slg. 1963, 1 ff. (van Gend \& Loos); EuGH, Urteil v. 19.01. 1981, Rs. 8/81, Slg. 1982, 53 ff., hier Rn. 21 ff. (Becker), st. Rspr.; Herdegen, M.: Europarecht, 8. Aufl. München, 2006, § 9, Rn. 13 f.; Triantafyllou, D., a. a. O., 195. 
Kompetenzausübungsregel wirkt, das Tatbestandsmerkmal des Nicht-Beeinträchtigens hingegen subjektiv-rechtlichen Gehalt entfaltet.

Erforderlich ist im Rahmen des unionsrechtlichen Schutznormansatzes schließlich, dass die Rechtsschutz suchenden Petenten im Schutzbereich des subjektiven Rechts stehen. Dies ist wegen der Bezugnahme auf die mitgliedstaatliche Ebene in Art. 17 Abs. 1, 2 AEUV durch Untersuchung des nationalen Staatskirchenrechts zu bestimmen.

\section{c) „Achten“als Einrichtungsgarantie, ,nicht-beeinträchtigen“ als subjektives Recht?}

Vor diesem Hintergrund lässt sich auch die Doppelung der Tatbestandsmerkmale „Achten“ und „Nicht-Beeinträchtigen“ schlüssig erklären. Das Merkmal „Achten" weist einen objektiv-rechtlichen Gehalt auf. Es wirkt als negative Kompetenznorm, weil es den Handlungs- und Entscheidungsspielraum des europäischen Gesetzgebers begrenzt, wenn es durch einen Rechtssetzungsakt der EU zu einem Konflikt mit dem gewährleisteten Status der Religionsgemeinschaften kommt. Über das Merkmal des „Achtens“ verlangt Art. 17 AEUV eine Selbstbeschränkung des EU-Gesetzgebers bei der Schaffung von inzidentem Religionsrecht der EU. Mit diesem objektiv-rechtlichen Gehalt wirkt Art. 17 Abs. 1, 2 AEUV als Einrichtungsgarantie bezüglich des Status (i. S.d. Art. 17 AEUV) der Religionsgemeinschaften gemäß ihren jeweiligen mitgliedstaatlichen Rechtsordnungen.

Das Merkmal „Nicht-Beeinträchtigen“ weist demgegenüber subjektiven Gehalt auf und vermittelt Art. 17 Abs. 1 AEUV den Charakter eins grundrechtsähnlichen Rechts.

\section{d) Unterschiedliche Rechte für religiöse und weltanschauliche Gemeinschaften?}

Die oben gewonnene Interpretation könnte zu dem Dilemma führen, dass religiöse Gemeinschaften in den Genuss eines subjektiven Rechtes kommen können, weltanschauliche jedoch nicht, weil Art. 17 Abs. 1 AEUV für religiöse Gemeinschaften das Tatbestandsmerkmal des Nicht-Beeinträchtigens erwähnt, Art. 17 Abs. 1 AEUV dies jedoch für weltanschauliche Gemeinschaften nicht wiederholt. Nach dem Wortlaut ist dieses Ergebnis zweifelsfrei zulässig. Dennoch ist es m. E. nicht hinnehmbar. Vor dem Hintergrund des Gleichheitssatzes wäre es nur vertretbar, wenn religiöse und weltanschauliche Gemeinschaften etwas Ungleiches wären, oder wenn sie zwar etwas Gleiches wären, aber die Ungleichbehandlung sachlich gerechtfertigt wäre. Ersteres setzt eine zuverlässige Abgrenzung 
voraus, die bisher definitorisch nicht gelingt. Eine sachliche Rechtfertigung für die Ungleichbehandlung ist auch nicht erkennbar. Daher ist Art. 17 Abs. 2 AEUV erweiternd auszulegen und das Tatbestandsmerkmal des Nicht-Beeinträchtigens in diesen Absatz hinein zu interpretieren. Dies gelingt, indem man den Zusatz „in gleicher Weise“ nicht nur eng auf das Wort „achten“ bezieht, sondern umfassend auf den gesamten Abs. 2. Somit kommen ggf. auch weltanschauliche Gemeinschaften in den Genuss des subjektiven Rechtes der NichtBeeinträchtigung.

\section{e) Zusammenfassung}

Dem Merkmal ,achten“ in Art. 17 Abs. 1, 2 AEUV ist ein objektiv-rechtlicher Gehalt zu entnehmen, der sich in Gestalt einer negativen Kompetenzregelung bei der Schaffung inzidenten Religionsrechts durch die legislativ tätige EU auswirkt und der Norm den Charakter einer institutionellen Garantie zu Gunsten des Status der Religionsgemeinschaften vermittelt. Gleichzeitig ist mit Bezug auf das Tatbestandsmerkmal „nicht beeinträchtigen“ ein subjektiv-rechtlicher Gehalt herleitbar. Dieser kann sich auf Grund der Wirkungsstruktur der Norm nur auswirken in dem oben definierten Bereich, in dem eine einschlägige Kompetenz der EU ein Tätigwerden ermöglicht, das sich inzident auf den Status einer Religionsgemeinschaft auswirkt. Für weltanschauliche Gemeinschaften gilt in Folge einer erweiternden Auslegung des Abs. 2 dasselbe. Art. 17 Abs. 1, 2 AEUV bietet keine Bereichsausnahme für die gesamten Regelungen der nationalen Staatskirchenrechte, sondern schützt mit seinem Tatbestandsmerkmal „Status“ institutionelle Rechtspositionen und Struktur prägende Regelungen des nationalen Religionsrechts. Insgesamt ist der Ansatz der Norm daher eng. In ihrem Wirkungskreis ist sie aber ein effektives Instrument gegen eine Statusbeeinträchtigung.

\section{Zusammenspiel mit Art. 10 Abs. 1 der Europäischen Grundrechtecharta}

Fraglich ist, wie die Religionsfreiheit des Art. 10 Abs. 1 EGRC und Art. 17 Abs. 1, 2 AEUV miteinander zusammenwirken. Diese Frage stellt sich, da gemäß Art. 6 Abs. 1 EUV die Charta der Grundrechte dieselbe Rechtsverbindlichkeit wie die Verträge haben soll. Im Verfassungskonvent herrschten dazu wie gezeigt unterschiedliche Auffassungen. Manche Delegierte meinten, Art. I-52 Abs. 1, 2 sei lediglich eine Wiederholung des Schutzes aus Art. 10 Abs. 1 EGRC und daher redundant, andere gingen von einer notwendigen Ergänzung aus. In der neuen 
deutschsprachigen Literatur wird der Kirchenerklärung neben der Struktur sichernden Funktion eine interpretationsverstärkende Wirkung im Zusammenhang mit Art. 10 Abs. 1 EGRC zugeschrieben, die in der Funktion einer Auslegungsmaxime den Gewährleistungsbereich der Religionsfreiheit erweitere und sich insbesondere zu Gunsten der korporativen Dimension des Grundrechts auswirke. ${ }^{69}$ Spricht man dem Kirchenartikel nach dem In-Kraft-Treten als Art. 17 AEUV - wie oben ausgeführt - auch eine subjektiv-rechtliche Dimension zu, reicht seine Bedeutung im Zusammenspiel mit der unionsrechtlichen Religionsund Weltanschauungsfreiheit noch über die einer Auslegungsmaxime hinaus. Die beiden Normen ergänzen sich und bilden komplementäre Bestandteile eines europarechtlichen Schutzkonzeptes für Religionsgemeinschaften.

Beide Bestimmungen weisen denselben Normbezug auf: Sie bieten den Religions- und Weltanschauungsgemeinschaften Schutz vor Ingerenzen durch Rechtsakte der Europäischen Union. Dabei wirkt Art. 10 Abs. 1 EGRC vom unionsrechtlichen Ausgangspunkt in die Sphäre nationalen Rechts hinein (Art. 51 Abs. 1 EGRC; bei der Durchführung des Rechts der Union), während Art. 17 Abs. 1, 2 AEUV nationale Normvorgaben in das EU-Recht hineinwirken lässt.

Beide Normen wählen einen grundrechtlichen Schutzansatz, der sich über einen persönlichen und einen sachlichen Schutzbereich erschließen lässt. Die Inhalte dieser Schutzbereiche sind jedoch jeweils unterschiedlich definiert. Für den persönlichen Schutzbereich bestimmt Art. 10 Abs. 1 EGRC autonom europarechtlich, welche Rechtssubjekte Träger des Grundrechts sind. Art. 17 AEUV rekurriert dafür auf die nationalen staatskirchenrechtlichen Systeme. Im sachlichen Schutzbereich ist das Bild ähnlich: Art. 10 Abs. 1 EGRC hat einen autonom europarechtlichen Bedeutungsgehalt (der sich zum Teil mittelbar aus den gemeinsamen nationalen Verfassungsüberlieferungen ergibt) und bietet genuin europarechtlichen Schutz. Art. 17 Abs. 1, 2 AEUV macht dagegen nationale materielle Regelungen zum Schutzobjekt vor europarechtlichen Rechtsakten und entwickelt seinen Schutzgehalt daher über vorgefundene nationale Regelungen.

Daher sind die Schutzbereiche der beiden Normen nicht deckungsgleich. Sie lassen sich vielmehr wie zwei Kreise beschreiben, die sich teilweise überschneiden. Somit ergibt das Zusammenwirken von Art. 10 Abs. 1 EGRC und Art. 17 Abs. 1, 2 AEUV ein unionsrechtliches Schutzregime mit drei Bereichen unter- 
schiedlichen Schutzes. ${ }^{70}$ Im ersten bietet allein das Grundrecht aus Art. 10 Abs. 1 EGRC den oben beschriebenen Schutz. Dieser enthält alle religionsgrundrechtlich relevanten Rechtsakte der Union, die den von Art. 17 Abs. 1, 2 AEUV erfassten Status der Religions- und Weltanschauungsgemeinschaften nicht berühren. Der Bereich hingegen, in dem nur Art. 17 Abs. 1, 2 AEUV seinen Schutz entfaltet, ist definiert als der Bereich außerhalb des Schutzbereichs von Art. 10 Abs. 1 EGRC, der durch den nationalrechtlichen Status gemäß Art. 17 Abs. 1, 2 AEUV umrissen wird. Eine Überlagerung (Schnittmenge) tritt auf in dem Bereich, in dem sowohl der Schutzbereich des Art. 10 Abs. 1 EGRC als europäisches Grundrecht eröffnet als auch der nationale Status einer Religions- und Weltanschauungsgemeinschaft betroffen ist. Eine Religionsgemeinschaft kann sich im Bereich der Schnittmenge sowohl auf Art. 10 Abs. 1 EGRC als auch auf den Schutz aus Art. 17 Abs. 1, 2 AEUV i. V.m. ihrem nationalen Status berufen. Wegen der unterschiedlichen Schutzgehalte sind die Normen nicht redundant. Beide Normen wirken hier parallel und stehen somit im Verhältnis der Idealkonkurrenz. Der Verlauf der Trennungslinie zwischen dem Bereich des Art. 17 Abs. 1, 2 AEUV und Art. 10 Abs. 1 EGRC kann jedoch nur abstrakt beschrieben werden, da der Schutzbereich der institutionellen Garantie des Art. 17 Abs. 1, 2 AEUV (und der entsprechenden subjektiv-rechtlichen Unterlegung durch das Merkmal des „Nicht-Beeinträchtigens“) abhängig ist von dem nationalen Status einer Religionsgemeinschaft und dessen Umfang. Je nach nationalem Status einer Religionsgemeinschaft wächst oder schrumpft die Größe der Schnittmenge von Art. 10 Abs. 1 EGRC und Art. 17 Abs. 1, 2 AEUV. Der Schutzbereich des Art. 17 Abs. 1, 2 AEUV ist variabel in Abhängigkeit vom nationalen Status einer Religionsgemeinschaft. Seine Grenze und damit das Verhältnis von Art. 17 Abs. 1, 2 AEUV und Art. 10 Abs. 1 EGRC kann nur im Einzelfall konkret bestimmt werden. Tendenziell kann (mit der gebotenen Vorsicht angesichts der Brüchigkeit der Systemeinteilung) festgehalten werden, dass Art. 17 Abs. 1, 2 AEUV auf europäischer Ebene nur ein geringes $\mathrm{Ma}$ an Schutz bieten kann, wenn eine Religionsgemeinschaft in ihrem Heimatstaat in einem Trennsystem existiert, da dort der Status der Religionsgemeinschaft wenig mit ausgeprägten Statusrechten versehen ist. Religionsgemeinschaften, die aus einem Kooperationssystem stam-

70 Ein konkretes sekundärrechtliches Beispiel für das Zusammenwirken und Ineinandergreifen der unionsrechtlichen Religionsfreiheit (Art. 6 Abs. 2 EUV i. V.m. Art. 9 EMRK; Art. 10 I EGRC) und der Erklärung Nr. 11/Art. 17 AEUV bietet Art. 4 der Richtlinie 2000/78/EG des Rates vom 27.12. 2000 zur Festlegung eines allgemeinen Rahmens für die Verwirklichung der Gleichbehandlung in Beschäftigung und Beruf, ABIEU Nr. L 303/16 vom 02. 12. 2000. 
men, genießen über die Statusverlängerung des Art. 17 Abs. 1, 2 AEUV auf EUEbene einen ausgeprägteren Schutz. Auf das Verhältnis von Staatskirchen zu Art. 10 Abs. 1 EGRC ist oben bereits eingegangen worden, sie dürften durch Art. 17 Abs. 1, 2 AEUV einen umfangreichen Schutz geltend machen können, da bei ihnen die nationalen Statusrechte am ausgeprägtesten sind.

Somit ergibt sich, dass Art. 10 Abs. 1 EGRC und Art. 17 Abs. 1, 2 AEUV zueinander „variabel komplementär“ sind. Sie ergänzen sich in unterschiedlicher Weise, abhängig vom nationalrechtlich prädeterminierten Status einer Religionsgemeinschaft, der sich über Art. 17 Abs. 1, 2 AEUV auf unionsrechtlicher Ebene auswirkt. Art. 17 Abs. 1, 2 AEUV ist somit nicht redundant und keine überflüssige Wiederholung des Art. 10 Abs. 1 EGRC, wie es vereinzelt im Verfassungskonvent vertreten worden ist. Vielmehr ist er eine wertvolle Ergänzung des Schutzes durch Art. 10 Abs. 1 EGRC, denn er leistet (wiederum in Abhängigkeit vom nationalen Status) die Ergänzung des grundrechtlichen Schutzes mit institutionellen staatskirchenrechtlichen Elementen, deren Fehlen bei Art. 9 Abs. 1 EMRK bereits Blum konstatiert hat. ${ }^{71}$ Blum warnte davor, diese Elemente quasi aus Wunschdenken in Art. 9 Abs. 1 EMRK hinein zu interpretieren. Mit Art. 17 Abs. 1, 2 AEUV gelingt ein Ansatz, der diese Anreicherung auch mit der gebotenen Sensibilität auf nationale Unterschiede leistet. Die unvermeidliche Kehrseite dieser Lösung ist es, dass auf EU-Ebene keine einheitliche Schutzintensität aller Religionsgemeinschaften besteht. Die Schutzintensität bleibt mit Blick auf Art. 17 Abs. 1, 2 AEUV vom jeweiligen nationalen Status der Religionsgemeinschaft abhängig.

\section{Die Dialogverpflichtung des Art. 17 Abs. 3 AEUV}

Gemäß Art. 17 Abs. 3 AEUV pflegt die Union mit den in Art. 17 Abs. 1, 2 AEUV genannten Organisationen einen offenen, transparenten und regelmäßigen Dialog in Anerkennung ihrer Identität und ihres besonderen Beitrages. ${ }^{72}$ Diese Norm ist neu in das Primärrecht der EU aufgenommen worden und ohne Vorläufer. Sie findet sich nicht in der Erklärung Nr. 11 zum Vertrag von Amsterdam.

71 Blum, N.: Die Gedanken-, Gewissens- und Religionsfreiheit nach Art. 9 der Europäischen Menschenrechtskonvention, Berlin, 1990, $177 \mathrm{f}$.

72 Vgl. Muckel, S., a.a.O., 197 f.; zur praktischen Ausgestaltung des Dialoges vgl. Weninger, M.H., a. a. O., $330 \mathrm{ff}$. 


\section{Normcharakter}

Art. 17 Abs. 3 AEUV ist keine teilhaberechtliche Ausgestaltung der Absätze 1 und 2, der Religionsgemeinschaften ein individuelles Anhörungsrecht vermitteln könnte. Als spezielle Ausformung des Prinzips der partizipativen Demokratie (Art. 8b EUV) gibt er einen objektiv-rechtlichen Maßstab für die Gestaltung eines Verfahrens vor (vgl. unten zum Tatbestandsmerkmal Dialog).

\section{Tatbestandsmerkmale}

\section{a) Kirchen und Gemeinschaften}

Zur Bestimmung der Berechtigten bezieht sich Art. 17 Abs. 3 AEUV auf die in Abs. 1, 2 genannten Organisationen. Daher gilt auch für Abs. 3, dass es sich hier nicht um autonome Begriffe des Unionsrechts handelt, sondern um Begriffshülsen, die gemäß dem jeweiligen nationalen Recht ausgefüllt werden müssen. Das bedeutet, dass der Zugang einer Organisation zum Dialog im Rahmen des Art. 17 Abs. 3 AEUV davon abhängig ist, ob sie nach ihrem nationalen Recht den Status einer religiösen oder weltanschaulichen Organisation innehat.

\section{b) Dialog}

Mit Art. 17 Abs. 3 AEUV werden die genannten religiösen Organisationen als Gesprächspartner der Union anerkannt. ${ }^{73}$ Art. 17 Abs. 3 AEUV ist vom Wortlaut und im Ansatz vergleichbar mit Art. 8b Abs. 2 EUV, der einen offenen, transparenten und regelmäßigen Dialog zwischen den Organen der Union und den repräsentativen Verbänden und der Zivilgesellschaft vorsieht. ${ }^{74}$ Art. $8 \mathrm{~b}$ Abs. 2 EUV stellt den Grundsatz der partizipativen Demokratie für die Union auf (vgl. auch den entsprechenden Art. I-47 VVE mit amtlicher Überschrift). Art. 17 Abs. 3 AEUV ist eine lex specialis zu Art. 8b EUV für den Dialog mit den Gemeinschaften im religiös-weltanschaulichen Bereich. Es handelt sich somit um eine spezialgesetzliche Ausformung des Grundsatzes der partizipativen Demokratie aus Art. 8b EUV.

$73 \mathrm{Zu}$ möglichen Zielen und Inhalten des Dialogs aus der Perspektive der Religions- und Weltanschauungsgemeinschaften vgl. Schnabel, P.R., $170 \mathrm{ff}$.

74 Rüfner, W., a.a. O., 766. 
Als Ausformung des Prinzips der partizipativen Demokratie ist die in Art. 17 Abs. 3 AEUV enthaltene Verpflichtung, einen Dialog zu führen, in institutionellem Sinne zu verstehen. Gemeint sind nicht individuelle Gespräche, sondern ein Dialog als Institution, d.h. im Sinne eines beständigen und geregelten Gedankenaustausches. Dieser nimmt vielfältige Formen an: ${ }^{75}$ Gespräche, Kongresse, gemeinsame Arbeitskreise (oder ad hoc zu spezifischen Anlässen), Konsultationen im Internet etc.

\section{c) Pflegen}

Selbstverständlich kann und darf die Union die Religionsgemeinschaften nicht verpflichten, an einer solchen Plattform des Austausches teilzunehmen. Art. 17 Abs. 3 AEUV enthält nur die Verpflichtung der Union, zu einem solchen Dialog bereit zu sein, ihn anzubieten, an ihm fördernd mitzuwirken und ihren Teil zum Zustandekommen eines solchen Austausches beizutragen.

Art. 17 Abs. 3 AEUV als Ausprägung der partizipativen Demokratie gibt keinen Anspruch im Sinne eines subjektiven Rechts einer einzelnen Religionsgemeinschaft, an einem bestimmten Treffen oder einer bestimmten Gesprächsrunde teilzunehmen, oder zu einer bestimmten Frage konsultiert zu werden. Wenn die Union allerdings solche Gespräche führt, so ist sie aus dem Diskriminierungsverbot (Art. 8 EUV, 21 f. EGRC) heraus verpflichtet, alle interessierten Religionsgemeinschaften gleich zu behandeln.

\section{d) Eigenschaften des Dialogs}

Der Dialog muss von der Union offen, transparent und regelmäßig gestaltet sein. Offen bedeutet, dass der Gedankenaustausch allen Religionsgemeinschaften im Sinne des Art. 17 Abs. 1, 2 AEUV zugänglich ist. ${ }^{76}$ Hier greift der Gleichheitsgrundsatz (Art. 8 EUV neu, Art. 21 f. EGRC). Der Gedankenaustausch darf nicht bestimmten Religionsgemeinschaften vorbehalten, sondern muss ein freies Angebot an alle sein. Weder Größe noch theologische Ausrichtung darf a priori ein Kriterium sein. Andererseits muss die EU die Möglichkeit haben, den Dialog effizient und effektiv zu gestalten. Kapazitätsbeschränkungen sind nach dem

76 Ebd., 331; weitergehend Naumann, K., a.a.O., 206, der dem Merkmal i.V.m. der Anerkennung der Religionen in der Präambel den - ohnehin selbstverständlichen - Auftrag entnehmen will, dass die EU den Dialog ernst nimmt und nicht lediglich „,als lästige Pflichtübung“ absolviert. 
allgemeinen Verhältnismäßigkeitsprinzip zu rechtfertigen. Die EU muss im Rahmen der Verhältnismäßigkeit für einen offenen Zugang zum Dialog sorgen; sie muss also nicht jedem interessierten Teilnehmer einen Platz bereit stellen (kein Anspruch auf Kapazitätserweiterung), aber sie muss für eine der Bedeutung der Veranstaltung angemessene Kapazität sorgen. Die Entscheidung über den $\mathrm{Zu}-$ gang zu Dialogveranstaltungen mit Kapazitätsbeschränkung muss auf der Grundlage formaler Kriterien (etwa Zeitpunkt der Anmeldung) ergehen, nicht auf Grund inhaltlicher Bewertung der Religion oder Weltanschauung. Der Zugang zu Dialogveranstaltungen muss allen Religionsgemeinschaften unter den gleichen Kriterien gewährt werden; eine Platzreservierung für große Religionsgemeinschaften ist m.E. auch nicht mit dem Hinweis gerechtfertigt, dass diese einen größeren Teil der Bevölkerung repräsentieren. Die Entscheidung über Gewicht und Bedeutung ist der neutralen EU vorenthalten; es obliegt den Religionsgemeinschaften, sich so zu organisieren, dass sie sich in den angebotenen Dialog einbringen können. Eine der demographischen Bedeutung angemessene Repräsentation der Religionsgemeinschaften wird sich dabei en passant ergeben, da größere Gemeinschaften über bessere Möglichkeiten der politischen Interessenvertretung verfügen. Rein formale Auswahlkriterien werden andererseits dafür sorgen, dass auch kleinen Religionsgemeinschaften der Zugang zu Dialogveranstaltungen offen steht und sie nicht von den großen verdrängt werden. Ein wichtiger Teil des gesamten Dialogs wird ohnehin im schriftlichen Verfahren stattfinden (Stellungnahmen, Internetkonsultationen), wo Kapazitätsgrenzen eine geringe Rolle spielen. Das Gebot der Offenheit gebietet es der EU, Dialogformen, deren Offenheit durch Kapazitätsgrenzen eingeschränkt ist, durch Alternativen mit geringeren Kapazitätseinschränkungen zu ergänzen. Konferenzen und Veranstaltungen mit begrenzter Teilnehmerzahl sollten etwa durch parallele Internetkonsultationen begleitet werden.

Transparent ist der Dialog, wenn er vom Öffentlichkeitsprinzip beherrscht wird. ${ }^{77}$ Die interessierten Kreise (sog. stakeholder) werden von den Veranstaltungen im Rahmen des Dialogs in Kenntnis gesetzt; die Inhalte des Gedankenaustausches sind für Dritte prinzipiell zugänglich (beispielsweise durch Einstellung in Datenbanken der EU im Internet). 
Regelmäßig heißt, dass der Gedankenaustausch unabhängig von spezifischen Anlässen stattfindet und somit nicht nur auf ad-hoc-Basis, sondern als beständiges Angebot konzipiert ist. ${ }^{78}$

\section{e) Anerkennung der Identität und des besonderen Beitrags}

Der Zusatz der Anerkennung der Identität und des besonderen Beitrags der genannten Organisationen bedeutet, dass die Union anerkennt, dass der Bereich des organisierten religiösen Lebens eine Sonderstellung einnimmt im Vergleich mit den anderen gesellschaftlich-politischen Akteuren, die sich im Dialog mit der Union befinden. Dies reflektiert das Bewusstsein der Union über die Tatsache, dass Religionsgemeinschaften sich ihrem Eigenverständnis nach in einer Rolle sehen, die das staatlich-weltliche Leben überschreitet. Damit stellt dieser Zusatz in Art. 17 Abs. 3 AEUV einen wichtigen Beleg dafür da, dass die EU Religion und ihre Organisationen in ihrer Eigenart dezidiert wahrnimmt und nicht (mehr) religionsblind ist. Keine Bedeutung hat die Formulierung hingegen für das politische Gewicht der Äußerungen, die die Religionsgemeinschaften im Rahmen des Dialogs tun. Dessen Bedeutung ist nicht über die Bedeutung des Dialogs mit anderen zivilgesellschaftlichen Akteuren hervorgehoben. Dies ist nicht zulässig, da die Union ein säkulares staatliches Gebilde ist, in dem religiös-weltanschauliche Ansichten nicht per se ein erhöhtes Gewicht haben.

Ferner wird die Auffassung vertreten, die Anerkennung der Identität bedeute, dass die Union die jeweilige Identität und Individualität der Religionsgemeinschaften zu berücksichtigen habe. ${ }^{79}$ Auch dies kann der Vorschrift entnommen werden. Die Union darf den Bereich des organisierten religiösen Lebens folglich nicht als undifferenzierte, homogene Größe begreifen und behandeln. Sie darf beim Dialog und der Berücksichtigung religiöser Belange keine „Zwangsökumene" organisieren, etwa indem sie davon ausgeht, dass eine protestantische Religionsgemeinschaft andere protestantische Gemeinschaften mitrepräsentiert.

Die Anerkennung des besonderen Beitrags unterstreicht die spezifische Rolle, die den Religionsgemeinschaften auf Grund ihres Selbstverständnisses eingeräumt wird. Ihr religiöser Beitrag zur Errichtung des geeinten Europas wird mit dieser Formulierung honoriert; gleichzeitig wird damit verdeutlicht, warum der

78 Ebd., 331.

79 Robbers, G.: Eine neue Verfassung für die Europäische Union, in: ZSE, 1/3 (2003), 383-399, hier 396 f.; ders.: Religion in Europa, a. a. O., $13 \mathrm{f}$. 
Dialog mit den Kirchen eine eigene Erwähnung neben dem Dialog mit der Zivilgesellschaft erhalten hat.

\section{Bewertung des Art. 17 Abs. 3 AEUV}

Die ersten Bewertungen der Dialogverpflichtung fallen bereits kontrovers aus. Eine Ansicht meint, dass Art. 17 Abs. 3 AEUV den Religionsgemeinschaften eine ungünstige Sonderrolle innerhalb des zukünftigen Gefüges der partizipativen Demokratie in der EU zuweise. ${ }^{80}$ Es handele sich um eine Ausklammerung des Religionsdialogs aus dem allgemeinen Mechanismus der partizipativen Demokratie, die im Dialog mit der Zivilgesellschaft (Art. 8b Abs. 2 EUV/Art. I-47 VVE) ihre Gestalt annimmt. Diese Ausklammerung reduziere die Bedeutung der Religionsgemeinschaften als Dialogpartner und dränge diese an den Rand des „Demokratischen Lebens der Union“" (so der Titel VI des ersten Teils des VVE). Der allgemeine Dialog gemäß Art. 8b EUV bzw. I-47 VVE enthalte nicht nur eine Dialogverpflichtung, sondern auch die Vorgabe, umfangreiche Konsultationen (Art. 8b Abs. 3 EUV/Art. I-47 Abs. 3 VVE) durchzuführen. Dies fehle in Art. 17 Abs. 3 AEUV/I-52 Abs. 3 VVE. Dieser Nachteil werde nicht aufgewogen durch einen vagen Hinweis auf eine nutzlose Anerkennung eines „,besonderen Beitrages“. Außerdem stehe Art. 17 Abs. 3 AEUV/I-52 Abs. 3 VVE in einem offensichtlichen Widerspruch zu den ersten beiden Absätzen des Artikels. Da Abs. 1, 2 die traditionellen Inhalte des Staatskirchenrechts dem nationalen Recht zuweise, sei unklar, was der Gegenstand des Dialogs unter Art. 17 Abs. 3 AEUV/I-52 Abs. 3 VVE sein solle.

Die Gegenmeinung sieht in der Sonderstellung des Dialogs mit den Religionsgemeinschaften keinen Ausdruck einer beabsichtigten Laizität der EU, sondern eine positive Anerkennung der tatsächlichen Besonderheiten. ${ }^{81}$ Religionsgemeinschaften seien nicht Verbände unter Verbänden und nicht lediglich partikulares Interesse unter anderen partikularen Interessen. Die besondere Erwähnung sei

80 Broglio, F. M.: Religious Aspects of the Constitution, in: Ferrari, S. (Hg.): God in the European Constitution, Supplement to the European Consortium for Church and State Research Newsletter, 4/2004, IV f.

81 Robbers, G.: Religion in Europa, a.a.O., 13; vgl. auch die Bewertung des VVE durch die COMECE: COMECE (Hg.): Vertrag über eine Verfassung für Europa. Elemente für eine Evaluierung, 11.03. 2005, http://www.comece.org/upload/pdf/pub_const_treaty_050311_DE.pdf, 16, 22, die in Art. I-52 Abs. 3 VVE ein Zeugnis der freundlichen und positiven Einstellung der Union gegenüber den Kirchen sieht, und die als Themen des Dialogs beispielsweise Wirtschaft und Soziales, Außen- und Sicherheitspolitik, Asyl und Migration, Bildung und Kultur sowie den Schutz der Religionsfreiheit in ihrer korporativen Dimension vorschlägt. 
folgerichtiger Ausdruck einer Besonderheit im Vergleich zu säkularen gesellschaftlichen Organisationen.

Die letztgenannte Ansicht erscheint zu sehr getragen von einer Selbstgewichtung der Religionsgemeinschaften und ihrer besonderen Beiträge und scheint die besondere Bedeutung des Religionsdialogs mit selbst empfundenen theologischen Bedeutungsansprüchen begründen zu wollen. Eine Verobjektivierung der subjektiven Einschätzung der Daseinsberechtigung der Religionsgemeinschaften darf das säkulare Recht der EU jedoch nicht vornehmen. Deshalb lässt sich mit solcherart Überlegungen die Sonderrolle des Art. 17 Abs. 3 AEUV nicht begründen, und auf diese Weise gewinnt sie auch keinen besonderen Wert. Doch erscheint auch der Pessimismus der ersten Ansicht nicht angebracht. Art. 17 Abs. 3 AEUV ist in der Tat eine lex specialis gegenüber dem allgemeinen Dialog gemäß Art. 8b EUV. Die Sonderrolle lässt sich mit rein weltlichen, der EU zustehenden Erwägungen begründen. Religionsgemeinschaften unterscheiden sich von den Verbänden der Zivilgesellschaft dadurch, dass sie ihre Ziele aus theologischen, nur subjektiv begründbaren Auffassungen herleiten. Dadurch unterscheidet sich oft auch die Art ihrer Willensbildung von derjenigen weltlicher Verbände. Sie vertreten Positionen, die sich nicht in Partikularinteressen erschöpfen, sondern stellen ihre Willensbildung in einen umfassenden Bedeutungskontext. Dessen vollständige Bedeutung erschließt sich zwar nur ihnen subjektiv, doch überträgt sich diese Tatsache in das weltlich wahrnehmbare Verhalten und Auftreten der Religionsgemeinschaften wie auch in die von ihnen vorgebrachten Beiträge und Ansichten. Im Rahmen der Grundsätze der Neutralität, Parität und Toleranz steht es der EU frei, diese faktisch wahrnehmbare Sonderrolle der Religionsgemeinschaften im Unionsrecht zu reflektieren. Praktisch bedeutet dies beispielsweise, dass den Religionsgemeinschaften deswegen eine besondere Rolle zuerkannt wird, weil sie etwa als ethisch-moralische Instanzen auftreten und respektiert werden, und die EU dies anerkennt, weil sie sich davon eine Bereicherung des öffentlichen Lebens verspricht. Eine so begründete Sonderrolle ist auch keine nutzlose Sonderbehandlung, sondern kann für die Gesellschaft fruchtbringend gestaltet werden.

Art. 17 Abs. 3 AEUV bedeutet für die Religionsgemeinschaften auch keine faktische Benachteiligung wegen einer fehlenden Konsultationsklausel in der Art des Art. 8b Abs. 3 EUV/Art. I-47 Abs. 3 VVE. Art. 17 Abs. 3 AEUV steht nicht in einem Exklusivitätsverhältnis zu Art. 8b Abs. 3 EUV. Sinn und Zweck der lex specialis ist, die soeben begründete Sonderrolle zu honorieren. Daher ist es sinnwidrig, Art. 17 Abs. 3 AEUV als Beschränkung oder Benachteiligung der 
Religionsgemeinschaften auszulegen. Art. 8b Abs. 3 EUV bleibt daher neben Art. 15 Abs. 3 AEUV anwendbar. Religionsgemeinschaften sind daher im Rahmen des Art. 8b Abs. 3 EUV zu konsultieren, wenn die allgemeinen Voraussetzungen einer Konsultation gegeben sind. ${ }^{82}$

Schließlich steht Art. 17 Abs. 3 AEUV auch nicht im Widerspruch zu den Absätzen 1 und 2. Diese weisen zwar die Statusangelegenheiten der Religionsgemeinschaften der Sphäre der Mitgliedstaaten zu. Doch bleiben vielfältige Gegenstände für den Dialog der EU mit den Religionsgemeinschaften. All die Berührungspunkte des ,inzidenten Religionsrechts“ der EU sowie gerade die diffizilen Abgrenzungsfragen zwischen den Kompetenzbereichen von EU und Mitgliedstaaten sind potentielle Gegenstände des Dialogs. Hinzu kommen Diskussionen über ethisch-moralische Fragen - etwa über die Behandlung des Klonens von Embryonen im EU-Recht -, die die Sonderstellung des Art. 17 Abs. 3 AEUV gerade ermöglichen und begünstigen soll. An möglichen Themen für Art. 17 Abs. 3 AEUV dürfte kein Mangel herrschen. 